\title{
BeppoSAX spectroscopy of MR 2251-178: A test for ionized reflection in radio quiet $\mathrm{QSOs}$
}

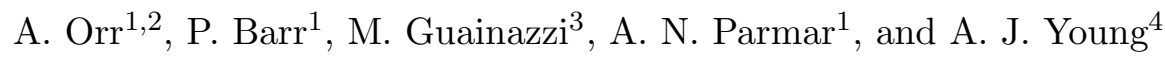 \\ 1 Astrophysics Division, Space Science Department of ESA, ESTEC, Postbus 299, 2200 AG Noordwijk, \\ The Netherlands \\ 2 Institut für Astronomie und Astrophysik Tübingen, Waldhäuser Str. 64, 72076 Tübingen, Germany \\ 3 XMM SOC, VILSPA, Villafranca del Castillo, Spain \\ 4 Department of Astronomy, University of Maryland, College Park, MD 20742, USA
}

Received 20 March 2000 / Accepted 9 July 2001

\begin{abstract}
We present the 0.1-200 keV BeppoSAX spectrum of MR 2251-178 observed at two epochs in 1998 separated by 5 months. Both epochs show identical spectral shape and X-ray flux. Analysis of the combined spectra allow us to confirm the presence of the ionized Fe K $\alpha$ line detected by ASCA and to test the presence of reflection from ionized material. The best self-consistent spectral fit is obtained when including a contribution from a mildly ionized reflector $\left(\xi_{0.01-100} \sim 1625 \mathrm{erg} \mathrm{cm} \mathrm{s}^{-1}\right.$, i.e. $\left.U_{0.1-10} \sim 0.14\right)$ with a reflection fraction $R_{\text {ion }} \sim 0.11$. An exponential cut-off to the direct power-law continuum is then required at $E \sim 100 \mathrm{keV}$. The low energy spectrum is absorbed by ionized matter with a column density $N_{\mathrm{W}}=\left(8.1_{-1.2}^{+2.1}\right) \times 10^{21} \mathrm{~cm}^{-2}$ and an $\mathrm{X}$-ray ionization parameter $U_{0.1-10}=0.06 \pm 0.01$. The warm absorber column is slightly lower than that measured with ASCA. This change could be caused by bulk motion. The BeppoSAX absorber ionization parameter globally agrees with the " $U$ versus Flux" correlation found for MR 2251-178 with EXOSAT, Ginga and ASCA. This suggests that the absorber is in a state of instantaneous ionization equilibrium.
\end{abstract}

Key words. galaxies: active - galaxies: quasars: individual - X-rays: galaxies

\section{Introduction}

The large variety of observed spectral and temporal variability properties of AGN is at the origin of the complex AGN classification scheme. Attempts to find a simple explanation for the different classes have resulted in the "unified model" of AGN (Antonucci 1993), in which the viewing angle of the observer is the key parameter. AGN luminosity is an additional parameter which can directly influence the physical state of matter located in the innermost regions of the nucleus. However the role of AGN luminosity is at present still not well understood. For instance, recent X-ray studies are still inconclusive about the existence of any fundamental differences between Radio Quiet (RQ) QSOs and RQ Seyfert 1 galaxies (Sy 1). These two classes have often been regarded as high- and low-luminosity manifestations of a same phenomenon (e.g. Staubert \& Maisack 1996; Lawson \& Turner 1997). For example, $2-10 \mathrm{keV}$ power-law spectral slopes of low $-z$ RQ QSOs have been found to be similar to those of low luminosity Sy 1 s $(\Gamma \sim 1.9-2$, e.g. George et al. 2000;

Send offprint requests to: A. Orr, e-mail: aorr@astro.estec.esa.nl
Reeves \& Turner 2000). Variability and warm absorber properties appear to be comparable in RQ QSOs and Sy 1s (George et al. ibid.). However, the strong Compton reflection "hump" which is common in Sy 1s has not been clearly detected in luminous RQ QSOs (Williams et al. 1992; Lawson \& Turner 1997; Reeves et al. 1997; George et al. 2000; Reeves \& Turner 2000). An analysis of ASCA data for high $z$ RQ QSOs (Vignali et al. 1999) shows that the $2-30 \mathrm{keV}$ (source frame) spectra are well described by a single power-law. Nandra et al. (1997b) showed that Fe K $\alpha$ line emission in RQ QSOs is weaker than in Sy 1 s and that the line profile and centroid energy both seem to depend on the AGN luminosity. This trend has recently been confirmed by George et al. (2000). Several mechanisms have been proposed to explain the differences between RQ QSOs and Sy 1s. For instance relativistic beaming could enhance the underlying X-ray continuum emission but not the iron line, which is thought to arise by fluorescence from cold or hot reflecting material (Williams et al. 1992). Another interpretation is that the X-ray luminosity of the central source regulates the ionization state of the Compton scattering medium which is probably the accretion disk itself. As the luminosity increases the atoms 
in the disk become increasingly ionized. At high ionization states the iron line is emitted at higher energies and its flux may decrease (Nandra et al. 1997b and references therein; Matt 1998; Ross et al. 1999, hereafter RFY99). Ultimately, the optical depth for photo-electric absorption decreases to such an extent that the continuum flux $<30 \mathrm{keV}$ is mostly reflected from the ionized disk and no longer absorbed (Basko et al. 1974; RFY99). Narrow band X-ray observatories have failed to detect any "hard tails" in RQ QSOs and the reflection component in these sources may so far have been confused with the underlying continuum emission. However, at high X-ray energies the cut-off due to Compton recoil and Klein-Nishina effects may be detectable.

In this paper we present broad band BeppoSAX spectroscopy of a RQ QSO. We show that this source has an ionized iron line and exhibits a high energy cut-off, despite lacking a Compton reflection hump. We compare our data with the predictions of reflection models in order to test for the presence of an ionized accretion disk.

MR 2251-178 is a nearby $(z=0.064)$ X-ray bright RQ QSO $\left(L_{\mathrm{X}} \sim 10^{45} \mathrm{erg} \mathrm{s}^{-1}\right)$. Early X-ray observations of this source with the Einstein Observatory revealed both a soft excess and variable intrinsic absorption. This was the first detection of a warm absorber in an AGN (Halpern 1984). The source was later monitored by EXOSAT between 1983 and 1984 with a sequence of 15 observations (Pan et al. 1990). During this period the $2-10 \mathrm{keV}$ flux varied between 1.8 and $3.3 \times 10^{-11} \mathrm{erg} \mathrm{s}^{-1} \mathrm{~cm}^{-2}$. The changes occurred on time-scales as short as $\sim 10 \mathrm{~d}$. In the same period the column density of the warm absorber also varied. Strong correlations between the soft excess flux, the warm absorber column density variability and the source luminosity were found. A subsequent reanalysis of the EXOSAT data together with Ginga observations (Mineo \& Stewart 1993) showed that the spectral behavior can be well described using a self-consistent warm absorber model, and that the ionization parameter of the gas is clearly correlated with the continuum flux. ASCA observations in 1993 and 1996 (Otani et al. 1997; Reeves et al. 1997) reveal a weak emission line at $6.57_{-0.07}^{+0.09} \mathrm{keV}$ due to partially ionized iron. In this case too, the ionization state of the warm absorber correlates well with the measured X-ray flux. High ionization absorption lines have been observed in the UV by Monier et al. (2001), these appear to be related to the X-ray warm absorber.

The BeppoSAX observations of MR 2251-178 extend the spectral range of previous X-ray spectroscopy of this object. At the same time they also expand the luminosity range of "warm-absorbed" AGN observed by BeppoSAX. A preliminary analysis of the warm absorber data is given by Orr et al. (2000).

\section{Observations}

We present results obtained with the Low-Energy Concentrator Spectrometer (LECS; 0.1-4 keV; Parmar et al. 1997), the Medium-Energy Concentrator
Spectrometer (MECS; 1.8-10 keV; Boella et al. 1997), the High Pressure Gas Scintillation Proportional Counter (HPGSPC; 4-120 keV; Manzo et al. 1997) and the Phoswich Detection System (PDS; 15-300 keV; Frontera et al. 1997) on-board BeppoSAX.

All these instruments are co-aligned and collectively referred to as the Narrow Field Instruments, or NFI. The MECS consists of two grazing incidence telescopes with imaging gas scintillation proportional counters in their focal planes. The LECS uses an identical concentrator system as the MECS, but utilizes an ultra-thin entrance window and a driftless configuration to extend the low-energy response to $0.1 \mathrm{keV}$. The non-imaging HPGSPC consists of a single unit with a collimator that remained on-source during the entire observation. The non-imaging PDS consists of four independent units arranged in pairs each having a separate collimator. Each collimator was alternatively rocked on- and off-source during the observation.

The region of sky containing MR 2251-178 was observed by BeppoSAX at two epochs: from 1998 June 14 17:58 UT to June 18 05:33 UT and from 1998 November 12 21:23 UT to November 16 05:32. Good data were selected from intervals when the elevation angle above the Earth's limb was $>4^{\circ}$ and when the instrument configurations were nominal, using the SAXDAS 2.0.0 data analysis package. The standard PDS collimator dwell time of $96 \mathrm{~s}$ for each on- and off-source position was used together with a rocking angle of $210^{\prime}$. LECS and MECS data were extracted centered on the position of MR 2251-178 using radii of $8^{\prime}$ and $4^{\prime}$, respectively. The June exposures in the LECS, MECS, HPGSPC, and PDS instruments are $60.3 \mathrm{ks}, 82.7 \mathrm{ks}, 53.3 \mathrm{ks}$, and $41.1 \mathrm{ks}$, respectively. The November exposures are in the same order $30.5 \mathrm{ks}, 61.2 \mathrm{ks}$, $13.9 \mathrm{ks}$, and $35.2 \mathrm{ks}$.

Background subtraction for the LECS and MECS were performed using the standard background files. For the HPGSPC it was carried out using data obtained when the instrument was observing the dark Earth. Finally the PDS background was estimated from the offset field according to the standard procedure.

\section{Results}

\subsection{Light curves and variability}

We have examined the LECS $0.1-2 \mathrm{keV}$ and the MECS 2-10 keV light curves for the 1998 June and November observations as well as the corresponding hardness ratios. The data were binned into intervals of $10 \mathrm{ks}$. The count rates and the hardness ratios are both relatively similar at the two epochs. The 1998 June light curves are well fit with a constant count rate. This gives, respectively, for the soft and hard X-ray bands $0.173 \pm 0.003$

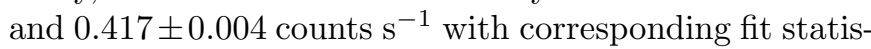
tics $\chi_{\nu}^{2}=1.1$ and 0.9 (degrees of freedom, d.o.f. $=29$ ). Fits to the November data give $0.183 \pm 0.004$ and $0.433 \pm$ 0.005 counts $\mathrm{s}^{-1}$, respectively, with $\chi_{\nu}^{2}=1.5($ d.o.f. $=24)$ and $\chi_{\nu}^{2}=3.4$ (d.o.f. $=21$ ). The poor statistics of the 

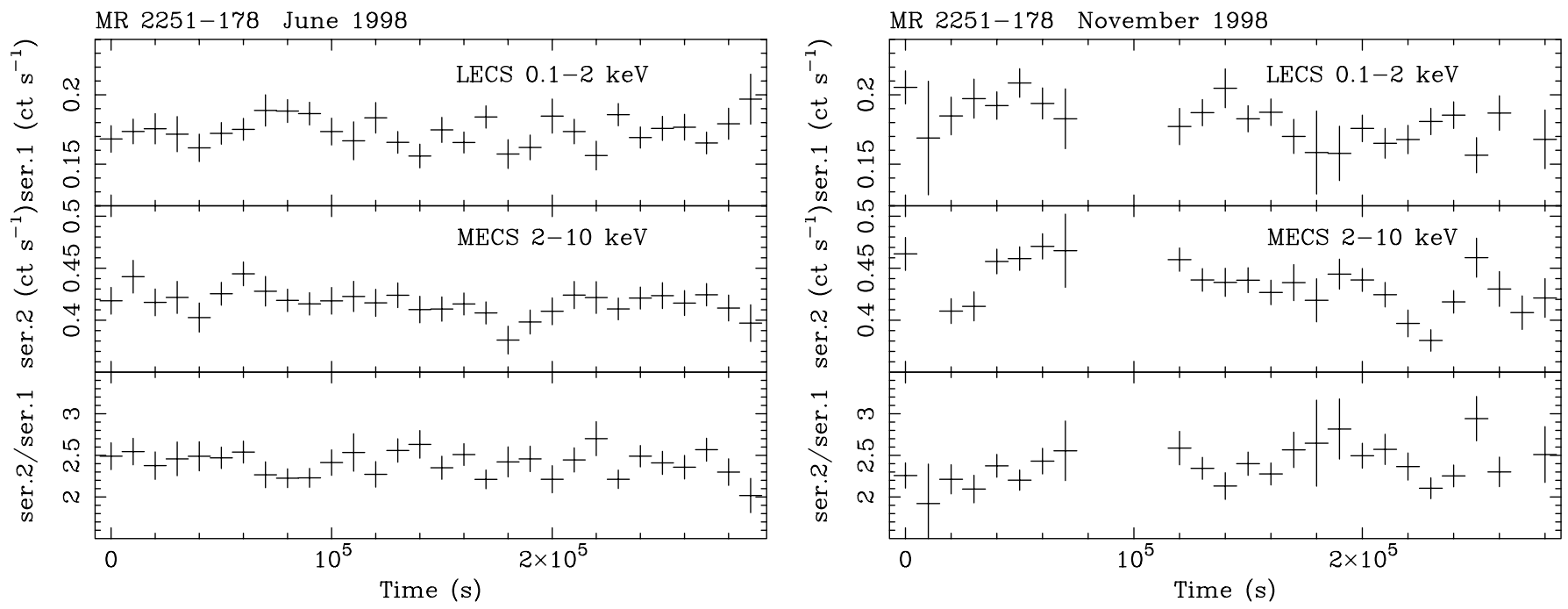

Fig. 1. $0.1-2 \mathrm{keV}$ and $2-10 \mathrm{keV}$ light curves and hardness ratios of the June and November 1998 BeppoSAX observations of MR 2251-178.

constant fits to the November data are an indication of variability. In fact, the November light curves are better fitted with slowly decreasing linear functions (minus $\sim 15 \%$ in 70 hours).

Finally, an interval of $30 \mathrm{ks}$ beginning $10 \mathrm{ks}$ after the observation start was ignored in the MECS November light curve. This time interval covers a drop in MECS count rate due to a change in pointing which could not be corrected by using the standard housekeeping data. The cleaned and linearized MECS November image shows what appears to be an extended, oblong source at the position of MR 2251-178. A comparision of light curves for different event extraction radii shows that this is an artefact caused by the displacement of the source in the MECS field of view. A similar displacement occurred in the LECS, however the available housekeeping data allow this effect to be corrected.

The 1998 June $2-10 \mathrm{keV}$ flux is $\left(4.03_{-0.12}^{+0.20}\right) \times$ $10^{-11} \mathrm{erg} \mathrm{s}^{-1} \mathrm{~cm}^{-2}$ (MECS) and the November flux is $(4.19 \pm 0.42) \times 10^{-11} \mathrm{erg} \mathrm{s}^{-1} \mathrm{~cm}^{-2}$.

\subsection{Spectral fits and interpretation}

The June and November spectra of MR 2251-178 were investigated both individually and as a single, summed spectrum by simultaneously fitting data from all the BeppoSAX NFI with the help of the XSPEC 11.01 spectral fitting package. The LECS and MECS spectra were rebinned to oversample the full width half maximum $(F W H M)$ of the energy resolution by a factor 3 and to have, additionally, a minimum of 20 counts per bin to allow use of the $\chi^{2}$ statistic. The HPGSPC spectrum was rebinned to have a minimum of 40 counts per bin and the PDS data were rebinned using the standard logarithmic binning recommended for this instrument. Data were selected in the energy ranges $0.1-4.0 \mathrm{keV}$ (LECS), 1.8$10 \mathrm{keV}$ (MECS), 8.0-12 keV (HPGSPC), and 15-200 keV

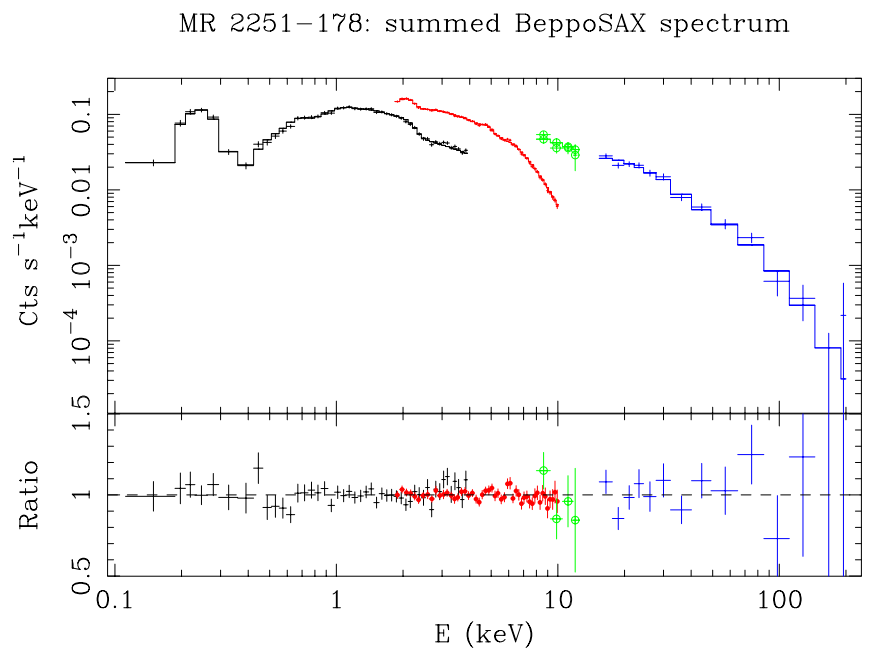

Fig. 2. The summed June and November 1998 BeppoSAX spectra of MR 2251-178. The MECS data are represented by small filled squares, the HPGSPC data by open circles. The fit model is RFY99 with a warm absorber.

(PDS) where the instrument responses are well determined and sufficient counts obtained. The photo-electric absorption cross sections of Morrisson \& McCammon (1983) and the solar abundances of Anders \& Grevesse (1989) are used throughout.

Factors were included in the spectral fitting to allow for flux normalization uncertainties between the instruments. For all instruments factors were constrained to be within their usual ranges during the fitting (see Fiore et al. 1999). In this paper all uncertainties are $90 \%$ confidence intervals for one parameter of interest, unless stated otherwise.

Because of the lower exposure times during the November 1998 observation the uncertainties on the fitted parameters are slightly larger than for those of the June spectrum. However, our fit results indicate that absolutely no significant spectral change occurred between the two epochs which are separated by $\sim 5$ months. All the fit 
Table 1. Fits to the summed June and November 1998 spectrum. The fitted energy ranges for the different instruments are 0.1-4 keV (LECS), 1.8-10 keV (MECS), 8-12 keV (HPGSPC) and 15-200 (PDS). All models include galactic neutral absorption $N_{\mathrm{H}}=2.78 \times 10^{20} \mathrm{~cm}^{-2}$ and, apart from the simple power-law (PL), also 3 absorption edges. The edge parameters are only listed for the cut-off power-law (COPL). All energies are given in the source rest-frame. The pexriv model is composed of both the reflected and the direct component. In this model the temperature of the ionized reflector has been fixed at $10^{6} \mathrm{~K}$. The parameters without uncertainty intervals were frozen during the fit. The ionization parameter is defined as in RFY99, integrating over the energy range $0.01-100 \mathrm{keV}$. The units of the ionization parameter $\xi$ are $\operatorname{erg~s}^{-1} \mathrm{~cm}$.

\begin{tabular}{|c|c|c|c|c|c|c|}
\hline model & $\Gamma$ & $\begin{array}{l}E_{\mathrm{FeK} \alpha}, E_{\text {cut }} \\
\mathrm{keV}\end{array}$ & $\begin{array}{l}E W \\
\mathrm{eV}\end{array}$ & $\begin{array}{l}E_{\text {Edge }} \\
\mathrm{keV}\end{array}$ & $\tau$ & $\chi_{\nu}^{2}$ (d.o.f.) \\
\hline PL & $1.61 \pm 0.01$ & & & & & $4.23(162)$ \\
\hline $\mathrm{PL}+\mathrm{FeK} \alpha$ & $1.67 \pm 0.01$ & $6.54_{-0.10}^{+0.11}$ & $89 \pm 24$ & & & $1.22(156)$ \\
\hline \multirow[t]{3}{*}{$\mathrm{COPL}+\mathrm{FeK} \alpha$} & $1.63 \pm 0.02$ & $6.54_{-0.11}^{+0.12}$ & $79 \pm 24$ & 0.74 & $0.59_{-0.14}^{+0.15}$ & $0.94(155)$ \\
\hline & & $133_{-35}^{+64}$ & & 0.87 & $0.33_{-0.13}^{+0.12}$ & \\
\hline & & & & $1.32_{-0.07}^{+0.08}$ & $0.20_{-0.05}^{+0.06}$ & \\
\hline model & $\Gamma$ & $E_{\mathrm{FeK} \alpha}, E_{\mathrm{cut}}$ & $E W$ & $R$ & $\xi$ & $\chi_{\nu}^{2}$ (d.o.f.) \\
\hline pexrav $+\mathrm{FeK} \alpha$ & $1.63 \pm 0.02$ & $6.54_{-0.11}^{+0.13}$ & $73_{-23}^{+24}$ & $<0.38$ & & $0.93(154)$ \\
\hline pexriv $+\mathrm{FeK} \alpha$ & $1.62_{-0.02}^{+0.01}$ & $\begin{array}{l}6.53_{-0.12}^{+0.14} \\
115^{+38}\end{array}$ & $62_{-25}^{+12}$ & $0.16_{-0.09}^{+0.15}$ & $465.1_{-240}^{+2003}$ & $0.89(153)$ \\
\hline RFY99 & $1.58 \pm 0.03$ & $\begin{array}{l}115_{-30} \\
102_{-26}^{+39}\end{array}$ & & $0.11_{-0.05}^{+0.06}$ & $1625_{-930}^{+1422}$ & $0.94(155)$ \\
\hline
\end{tabular}

PEXRIV confidence contours

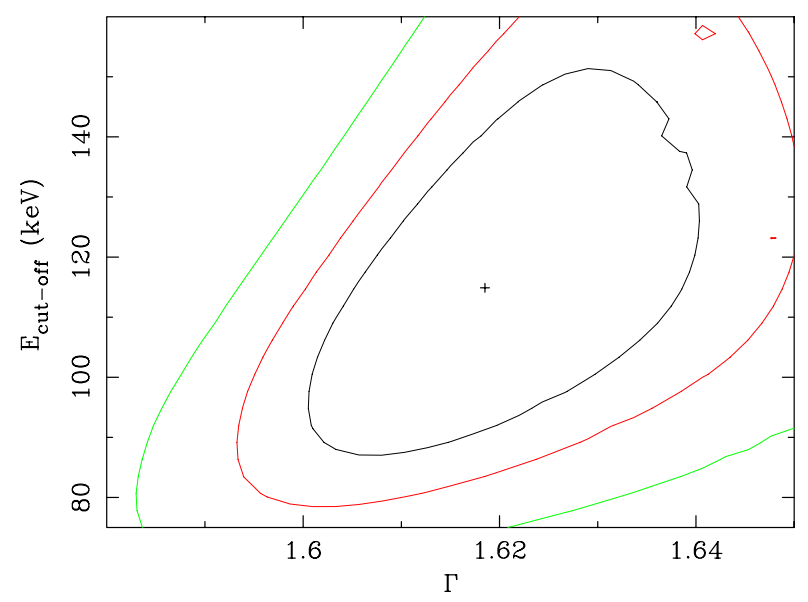

Fig. 3. Contour plot of the parameters $E_{\text {cut-off }}$ and $\Gamma$ in the ionized reflection model pexriv. The contours indicate 68, 90 and $99 \%$ confidence levels.

parameters for the individual spectra are compatible within the statistical uncertainties for any of the models tested. For this reason and because the observed fluxes in June and November are nearly identical with no large structure change in the light curves we have summed the spectra for the two epochs in order to obtain a higher signal to noise ratio.

We tried fitting the summed continuum with several models (see Table 1), starting with a power-law which gives a totally unacceptable fit $\left(\chi_{\nu}^{2}=4.2\right.$, d.o.f. $\left.=162\right)$. Strong deviations appear in the ranges $E \sim 0.4-3 \mathrm{keV}$ and at $E \gtrsim 80 \mathrm{keV}$ as illustrated in Fig. 4. The spectral complexity in the soft X-rays is due to the warm absorber and is discussed further on. At high energies the data points

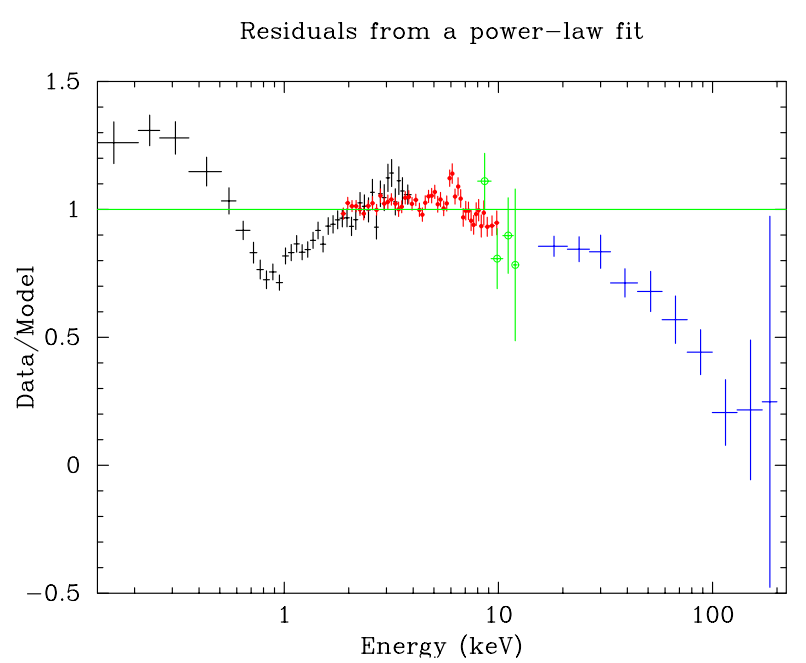

Fig. 4. Data-to-model ratio for a simple power-law with galactic neutral absorption. The MECS data are shown with solid squares and the HPGSPC with open circles. The warm absorption and the high energy cut-off are evident.

clearly fall below a simple power-law. Highly significant improvement is brought with an exponential high energy cut-off to the power-law at $E_{\text {cut-off }}>70 \mathrm{keV}$, (the confidence level of the improvement is $>99.9 \% ; F$-statistic $F>40$ for $\Delta$ d.o.f. $=1$, with respect to a simple powerlaw, with or without an Fe K $\alpha$ line or a warm absorber; the value of the cut-off energy is discussed below). However, as described in the following sections, the best fit to the summed 0.1-200 keV BeppoSAX spectrum of MR 2251178 is obtained with an ionized reflection model. Such a model predicts a drop in high X-ray flux but, unlike the case of reflection onto cold matter, a weaker or absent "Compton hump". 


\subsubsection{Significance of the high-energy cut-off}

The knowledge of the spectral shape above $\sim 10 \mathrm{keV}$ is an important key to the interpretation of the present BeppoSAX data. Therefore we have taken special care in verifying the quality of the PDS data (for details about the PDS instrument see Frontera et al. 1997).

It has been shown (by Guainazzi \& Matteuzzi 1997) that the total systematics in the PDS 20-200 keV range due to background subtraction are lower than $0.026 \mathrm{~s}^{-1}$ (corresponding to approximately $5.8 \%$ of the PDS 20$200 \mathrm{keV}$ source count rate for MR 2251-178, which is $0.45 \mathrm{cts} \mathrm{s}^{-1}$ ). The observed flux of a one mCrab source with photon index $1.5-2$, is well above the systematics $(\gg 1 \sigma)$ up to $60 \mathrm{keV}$ and remains above the systematics $(\geq 1 \sigma)$ up to $100 \mathrm{keV}$. (MR 2251-178 has a flux of $\sim 3$ mCrab in the $20-200 \mathrm{keV}$ range).

We have also checked the consistency of the spectra measured by the two PDS half-arrays (A \& B) which see the sky (source and background field) through two different collimators. This was done by fitting either one of the PDS half-arrays (A \& B) spectra together with the LECS, MECS and HPGSPC data. Because the data were obtained during two epochs (June and November 1998), we first tested the half array data for June only and then for the summed June and November. This is justified because the PDS rocking collimator positions happen to coincide within 0.6 degrees. Using the "cut-off power-law + 3 edges $+\mathrm{Fe} \mathrm{K} \alpha$ line" model in Table 1 one finds that the cut-off energies are all compatible with those found using the combined PDS data (A + B half-arrays). Also, in all cases the presence of the cut-off is if significant at greater than the $99.5 \%$ confidence level using the F-statistic.

We have also tested whether the PDS so-called "spike" events can contribute to the PDS spectral shape. The answer is no: in our two observations of MR 2251-178 the contribution of the "spike" events to the PDS spectral shape is negligible.

However, PDS event filtering using the so-called "Variable Rise Time" (VRT) selection does bring a significant improvement to the quality of fit, by increasing the signal to noise ratio of the PDS data. Therefore this filtering has been applied to all our PDS data.

\subsubsection{The pexriv model}

Our results with a pexriv model including a warm absorber and an Fe K $\alpha$ line (see also Fig. 3) are the following: a power-law slope $\Gamma=1.62_{-0.02}^{+0.01}$, a high energy exponential cut-off of the incident power-law at $E_{\text {cut-off }}=$ $115_{-30}^{+38} \mathrm{keV}$, the temperature of the ionized reflector $T_{\text {disk }}$ being fixed at $10^{6} \mathrm{~K}$, an ionization parameter of the reflector $\xi=465_{-240}^{+2003} \mathrm{erg} \mathrm{cm} \mathrm{s}^{-1}$ and finally the scaling factor for reflection $R_{\text {ion }}=0.16_{-0.09}^{+0.15}$, which is in fact the solid angle $R=\Omega / 2 \pi$ subtended by the reflecting disk. It should be noted that, for these reflection models, we use $\xi$ as it is defined in RFY99, i.e. with the ionizing flux integrated between $0.01-100 \mathrm{keV}$. Because in the pexriv model $\xi$ is defined between $5-20 \mathrm{keV}$ we have made the necessary conversions, assuming a photon index $\Gamma=1.6$ (i.e., $\xi_{\text {RFY99 }} \sim \xi_{\text {pexriv }} \times 4.4$ ).

The fit statistics are good, with $\chi_{\nu}^{2}=0.89$ for 153 d.o.f. The low value of the scaling factor $R$ tells us that most of the flux in this model is still coming from the direct, non-reflected component. Furthermore, one should bear in mind that a moderate ionization parameter such as obtained with this model, still produces a significant reflection "hump". Since the present data obviously lack such a hump the scaling factor cannot be large. In all our reflection fits we fixed the inclination angle of the disk with respect to the observer so that $\cos i=0.45$. This was necessary because in our fits the viewing angle and the reflection scaling factor are not independent, nor are they well constrained with respect to one another at low values $(R \lesssim 0.3$ or $\cos i \lesssim 0.2$ ). In fact, in the present pexriv fit the entire range of numerically permitted $\cos i$ values, i.e. $\cos i=0.05-0.95$ lies within the $99 \%$ confidence interval for this parameter. At $\cos i \sim 0.05 R$ is poorly constrained with a lower limit value of $R>0.4$. At $\cos i \sim 0.95$ we have $0.05<R<0.22$. $R$ and $\cos i$ are linked in the fitting process because smaller values of $i$ produce stronger reflection humps and vice-versa.

\subsubsection{The pexrav model}

Reflection on cold matter (e.g. neutral Compton reflection model pexrav in XSPEC) gives, compared to pexriv, a slightly worse fit with $\chi_{\nu}^{2}=0.93$ for 154 . The difference is significant at the $99 \%$ confidence level. The neutral reflection scaling factor is weakly constrained with an upper limit $R_{\text {neut. }}<0.38$.

\subsubsection{A self-consistent ionized reflection model: RFY99}

The pexriv model in XSPEC does not include Fe K $\alpha$ emission and one therefore needs to add a separate Gaussian line component to the fit model. Because of this drawback we have also tested the self-consistent ionized reflection models published by Ross and collaborators (RFY99) which include $\mathrm{Fe} \mathrm{K} \alpha$ line emission and take into account the destruction of $\mathrm{K} \alpha$ photons by Auger effects. The models were specially computed for the values of the incident power-law slope observed in MR 2251-178. As with pexriv we set the iron abundance at the solar value and included a warm absorber. The RFY models do not include the "incident" continuum so it is necessary to add it separately, in this case an exponentially cut-off power-law. We hereafter refer to this combination of components as the "RFY99 model". The power-law slope of the incident continuum and the slope specifying the reflection component were fixed to have the same value. It should be noted that, unlike pexriv, the RFY99 reflection assumes a sharp, 
step-like cut-off $^{1}$ of the incident spectrum at exactly $100 \mathrm{keV}$. This causes the RFY99 reflection component to decline steeply above $\sim 50 \mathrm{keV}$. The RFY99 model gives a good a fit with $\chi_{\nu}^{2}=0.94$ for 155 d.o.f., see Fig. 2. If the incident spectrum is taken to be a power-law without an exponential cut-off the fit becomes significantly worse $\left(\chi_{\nu}^{2}=1.40\right)$. The "RFY99" reflected fraction is $\sim 0.11$ and is compatible with the pexriv value. Both models also give comparable high energy cut-offs at $\sim 100 \mathrm{keV}$. We have checked that this value is not an artefact caused by the sharp cut-off assumed in the RFY99 reflection component (at $E_{\text {rest }}=100 \mathrm{keV}$ and $E_{\text {obs }}=94 \mathrm{keV}$ ). Indeed, if a "hard tail" is added to the RFY99 reflection component, with an exponential cut-off at $200 \mathrm{keV}$, the fitted continuum cut-off energy remains at $\sim 100 \mathrm{keV}$ with a $90 \%$ confidence upper limit $E_{\text {cut }}<200 \mathrm{keV}$. The reason for this is most likely the relatively low reflection scaling factor, $R \sim 0.11$.

A noteworthy analogy can be seen between the pexriv and RFY99 models concerning the ionization parameter of the reflector. Due to non-linear $\chi^{2}$ space two distinct fits are possible with the RFY99 models. In the first case the ionization parameter is $\xi=1625_{-930}^{+1422} \mathrm{erg} \mathrm{cm} \mathrm{s}^{-1}$ and in the second $\xi<35.4 \mathrm{erg} \mathrm{cm} \mathrm{s}^{-1}$. This second value corresponds to a nearly neutral reflector and gives a worse fit than with the first value $\left(\Delta \chi^{2}=4.4\right)$. Such a behavior is analogous to the pexriv versus pexrav solutions and therefore gives support to the validity of the ionized reflection models and their fitted parameter values.

These reasons lead us to consider hereafter only the fit solution yielding the higher value of $\xi$. The ionization parameter measured with pexriv is poorly constrained making it difficult to compare with the RFY99 value.

The value of $\xi$ (with the RFY99 model +3 edges) is driven mainly by the two following factors: first, the intensity of the $\mathrm{Fe} \mathrm{K} \alpha$ line and second, the low energy $(<6.4 \mathrm{keV})$ spectral shape. This is verified by performing the fits over limited energy ranges. For instance, excluding the $5.5-8$ or $5-12 \mathrm{keV}$ range leads to significantly higher ionization parameters, and weaker $\mathrm{Fe}$ lines. Likewise, if the range below $5 \mathrm{keV}$ is excluded, then $\xi$ decreases significantly.

$\xi=1625$ implies a hot $\mathrm{Fe} \mathrm{K} \alpha$ line in the reflection component, with $E_{\text {rest }} \sim 6.8 \mathrm{keV}$ and $E_{\text {obs }} \sim 6.4 \mathrm{keV}$. This is "hotter" than the line energies found with the other, non-self-consistent, fit models in Table 1 . However, from Fig. 5 we see that the "hot" line is still consistent with the MECS data. Indeed, no large fit residuals appear at the Fe K $\alpha$ line with the RFY99 model.

Nevertheless, an improvement in $\chi^{2}$ can be brought to the global RFY99 fit if an additional $\mathrm{Fe} \mathrm{K} \alpha$ line $\left(E_{\text {rest }}=\right.$ $\left.6.53_{-0.14}^{+0.15} \mathrm{keV}, E W=55_{-11}^{+32} \mathrm{eV}\right)$ is included $\left(\chi_{\nu}^{2}=0.9\right.$, $\Delta \chi^{2}=8.8$ for $\Delta$ (d.o.f. $)=2$ ). But the model is then no

\footnotetext{
${ }^{1}$ As mentioned in the RFY99 paper, extending the illuminating spectrum to higher energies would raise the corresponding portion of the emergent spectrum via Compton downscattering of higher energy photons.
}

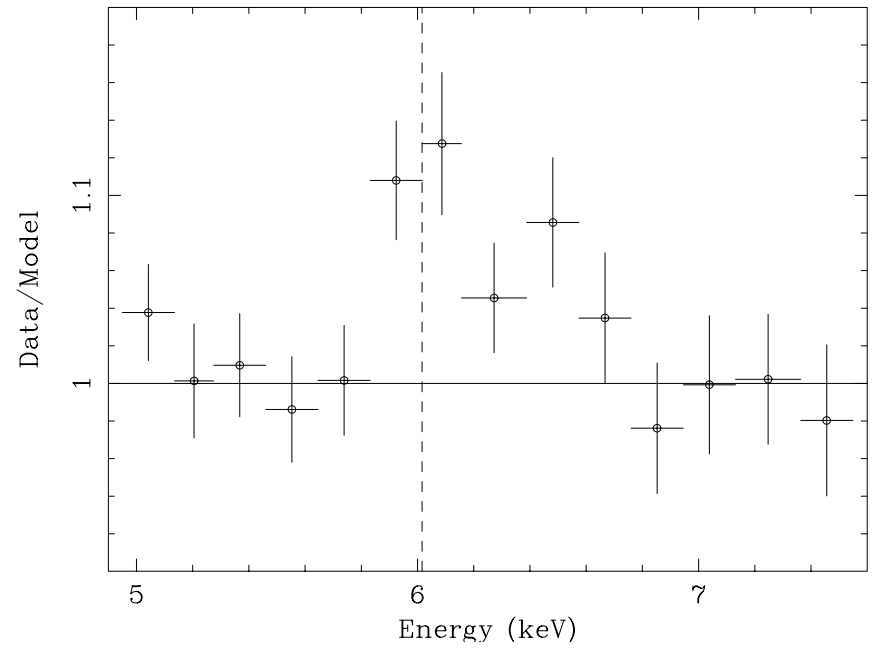

Fig. 5. Fe K $\alpha$ line profile: MECS data to model ratio in observer's reference frame. The model is a cut-off power-law with a Gaussian emission line for $\mathrm{Fe} \mathrm{K} \alpha$, where the line normalization has been set to zero for the plot. The energy of neutral $\mathrm{Fe} \mathrm{K} \alpha$ emission $(6.4 \mathrm{keV})$ is indicated by the dashed line.

longer self-consistent and, in order to "compensate" for the additional line, the ionization parameter of the reflector tends to increase and the reflected fraction to decrease. It may be a hint that a slightly different $\mathrm{Fe}$ abundance is required. For instance, increasing the Fe abundance increases both the line flux and the depth of the Fe K-edge. However, any iron over-abundance must be relatively low. This is because the ionization parameter derived from the RFY99 model indicates that the reflecting matter is in the regime where ions are already too highly ionized to permit Auger destruction of iron line photons. Therefore these photons escape from the reflector to produce the observed hot iron line (Fabian et al. 2000). Another possibility is that the line is due to neutral reflection from matter further out, e.g. the torus or a cold phase of the accretion disk.

\subsubsection{The Fe $\mathrm{K} \alpha$ line}

Fits of the $\mathrm{Fe} \mathrm{K} \alpha$ line (see Fig. 5) show that it is weak but very significant at $>99.9 \%$ confidence level (the $F$-test value for the presence of a line in the cut-off power-law model of Table 1 is $F=15.9$ for a difference of 2 d.o.f.). A narrow line fit gives $E_{\text {line }}$ (rest) $=6.53_{-0.12}^{+0.14} \mathrm{keV}$, an equivalent width $E W=62_{-25}^{+12} \mathrm{eV}$ (the continuum is here the best fit pexriv model). The line energy is consistent with ionization stages Fe XIII-XXIII. A significantly worse fit is obtained if one forces the line energy to be $6.4 \mathrm{keV}$ ( $>95 \%$ confidence level). In the fit models of Table 1 the width of the narrow line has been frozen at $\sigma=0.1 \mathrm{keV}$ because no there is no significant improvement in the fit statistics when leaving $\sigma$ free.

Nevertheless, we checked whether broad wings are present beneath the narrow core. We fitted the MECS continuum spectrum with a power-law continuum and 
galactic absorption to which we added, in turn, a narrow line or a "narrow plus broad Gaussian line". In the second case both lines were required to have the same centroid energy. There is no strong evidence for the presence of a broad Gaussian component of the Fe K line. Such a broad line is not required by the data, the confidence level for its inclusion being only $\sim 80 \%$, using the $F$-statistic. The resulting line width is $\sigma_{\text {broad }}=1.25_{-0.77}^{+2.22} \mathrm{keV}$. The equivalent widths of the core and the broad components are then 53.0 and $116.0 \mathrm{eV}$, respectively. So, we cannot completely rule out some broadening of the line by e.g. Compton scattering.

Likewise, we have performed fits with the XSPEC diskline model using the MECS data alone and compared them with the broad Gaussian line fits. The fit quality of either model is statistically equivalent. Therefore it is not possible to formally exclude the presence of a relativistically "blurred" line. The width of the peak line emission remains lower than the instrument's energy resolution at $6 \mathrm{keV}$ ( $~ 8 \%$ in the MECS).

When testing the diskline model we tried to minimize the amount of free parameters by estimating the outer radius of the disk. We considered the simple case of an annulus illuminated from one side and a non-rotating central black hole with a mass of $2.5 \times 10^{8} M_{\odot}$. This mass was derived for MR 2251-178 by Brunner et al. (1997) by fitting geometrically thin $\alpha$ accretion disc models. They also found an inclination of the disc of 70 (39-82) degrees. From the value of the ionizing X-ray flux and the ionisation parameter obtained with the RFY99 fit it is possible to estimate the surface area of the ionized disk, assuming a hydrogen number density of $n_{0} \sim 10^{15} \mathrm{~cm}^{-3}$. From the value of this surface area one can derive the outer radius of the disc $\left(\sim 6.46 R_{\mathrm{G}}\right)$, the inner radius being fixed at $6 R_{\mathrm{G}}$. The resulting diskline feature is double peaked, several keV broad and skewed. The blue peak is, however, about 4 times stronger in intensity than the red peak. And the $F W H M$ of the blue peak is $<0.1 \mathrm{keV}$ (not resolved by BeppoSAX).

In conclusion, our results with a narrow emission line confirm the previous measurements of the line made at higher energy resolution with ASCA $\left(E_{\text {line }}=\right.$ $6.57_{-0.07}^{+0.09} \mathrm{keV}, E W=62 \pm 32 \mathrm{eV}, \sigma<0.22 \mathrm{keV}$, Reeves et al. 1997) and they also are consistent with the $E W$ luminosity relation suggested by Iwasawa \& Taniguchi (1993) and confirmed by Nandra et al. (1997b.) This relation is a strong anticorrelation between the luminosity and the $E W$, an "X-ray Baldwin effect" which suggests Fe $\mathrm{K} \alpha$ line core $E W \mathrm{~s}$ lower than $\sim 80 \mathrm{eV}$ at $L_{2-10 \mathrm{keV}} \gtrsim$ $10^{45} \mathrm{erg} \mathrm{s}^{-1}$, which is the luminosity of MR 2251-178.

\subsubsection{The complex low-energy $\mathrm{X}$-ray spectrum}

A soft excess component is not required by the data. The ionized gas of the warm absorber can account for the spectral complexity below $2 \mathrm{keV}$. Likewise, we see that the best fitting models (which include a warm absorber) do not require excess cold absorption. The upper limit on excess neutral absorption is $0.16 \times 10^{20} \mathrm{~cm}^{-2}$, using the RFY99 model.

The soft X-ray spectrum of MR 2251-178 is complex and can be well fit by considering the effects of strong absorption by partly ionized matter. Below $1 \mathrm{keV}$ this absorption is essentially due to highly ionized oxygen. BeppoSAX cannot spectrally resolve the two oxygen edges $\mathrm{O}$ VII and $\mathrm{O}$ VIII at 0.74 and $0.87 \mathrm{keV}$ in the source rest frame. Therefore fits have been performed by multiplying the continuum model by either a single "blended" absorption edge or two oxygen edges with energies fixed at the values above. The "blended" edge parameters are: $E_{\text {edge }}=0.81 \pm 0.01 \mathrm{keV}, \tau=0.80 \pm 0.05$. In the second case, $\tau_{\mathrm{O} 7}=0.38 \pm 0.07$ and $\tau_{\mathrm{O} 8}=0.48 \pm 0.06$. A third edge brings further significant improvement to the fit statistics $(F$-statistic $F=21.41$ for $\Delta$ d.o.f. $=2)$. This third edge $(E \sim 1.32 \mathrm{keV}, \tau \sim 0.20)$ can be due to $\mathrm{Ne} \mathrm{X} \mathrm{K}, \mathrm{Mg}$ I-IV K or Ni XIV-XV L.

Alternatively, fits with warm absorber models were tested. The models were calculated using ION98, the 1998 version of the photo-ionization code ION (Netzer 1996). They were combined with a cut-off power-law continuum and a Gaussian Fe $\mathrm{K} \alpha$ line. The best fit gives a column density of the warm absorber $N_{\mathrm{W}}=\left(8.1_{-1.2}^{+2.1}\right) \times 10^{21} \mathrm{~cm}^{-2}$ and an oxygen ionization parameter $U_{\text {oxy }}=0.018_{-0.003}^{+0.004}$. The oxygen ionization parameter $U_{\text {oxy }}$ is the unitless ratio of ionizing photon flux to the electron density and is defined by integrating the rate of photon emission over the oxygen K-shell continuum, i.e. between $0.538-10 \mathrm{keV}$. In the present case $U_{\text {oxy }}$ translates to an X-ray ionization parameter (defined by integrating between $0.1-10 \mathrm{keV}$ ) $U_{0.1-10}=0.060_{-0.009}^{+0.012}$. The fit statistics with the warm absorber model are significantly better (at more than $90 \%$ confidence) than with the triple absorption edge fits: $\Delta \chi^{2}=5.13$ for $\Delta$ d.o.f. $=2$, leading to an $F$-statistic $F=2.9$.

We note that the ionization parameters of the reflection component and the warm absorber are similar: using the apropriate conversion and flux integration between $\xi_{0.01-100}$ and $U_{0.1-10}$ (e.g. Netzer 1996; George et al. $1998)$ the reflector has $U_{0.1-10}=0.04(0.02-0.22)$, with the pexriv fit and $U_{0.1-10}=0.14(0.06-0.27)$, with the RFY99 fit, respectively. But the warm absorber is optically thin and the RFY99 reflector optically thick. So, despite their similar ionization parameters they most likely are distinct.

The comparison of our warm absorber fit results with previous warm absorber results for MR 2251-178 is complicated by the different formalisms used in the literature to define the ionization parameter, in particular the integration range of the ionizing photon flux. For a start, we note that our measured warm absorber column, $N_{\mathrm{W}}=\left(8.1_{-1.2}^{+2.1}\right) \times 10^{21} \mathrm{~cm}^{-2}$ is consistent with values derived from Ginga and EXOSAT data (Mineo et al. 1993), even when MR 2251-178 was in weaker or brighter states than seen by BeppoSAX. An earlier study of the same 
EXOSAT data by Pan et al. (1990) seemed to suggest a column versus flux trend whereby the warm absorber column decreases with increasing X-ray flux. However, results from Mineo et al. (1993) as well as from Otani et al. (1997), for ASCA data, excluded such a trend. Unlike the EXOSAT data with relatively low statistics, the high signal to noise ASCA spectra indicate slightly lower values of $N_{\mathrm{W}}$ than the BeppoSAX spectra do $\left(\ll 7.5 \times 10^{21} \mathrm{~cm}^{-2}\right)$. Finally, the Ginga, EXOSAT and ASCA data all show a well defined correlation between the ionization parameter and the X-ray flux. Using the ionization parameter "translation" curves of George et al. (1998, Fig. 1) which give the relations between various definitions of the ionization parameter, $\xi, U$ and $U_{0.1-10}$, we estimate that the ionization parameter of the warm absorber in MR 2251-178 globally agrees with the EXOSAT, Ginga and ASCA " $U$ versus flux" correlation curves. To summarize, it seems that the warm absorber in MR 2251-178 is responding in a consistent way to the slow variations of the ionizing continuum flux and therefore is at most times in a state of ionization equilibrium. Any change in absorber column density between the 1996 ASCA and the 1999 BeppoSAX observations could be explained by bulk motion of the absorber material across our line of sight.

\section{Discussion}

Both the XSPEC pexriv and the RFY99 models give very good fits to the BeppoSAX spectrum of MR 2251-178. Of all the models we tested only the RFY99 model can by itself and in a self-consistent way account for the ionized Fe line, part of the high energy cut-off and the lack of reflection hump, however, this model assumes a reflector with constant density. The upper layers of the reflector, which are subject to strong external illumination probably have lower densities and higher effective ionization parameters than the inner layers. This would tend to suppress Fe $\mathrm{K} \alpha$ emission in the outer layers (RFY99). Nayakshin et al. (2000) and Ballantyne et al. (2001) have recently calculated models of reflection on an ionized atmosphere in hydrostatic equilibrium. Nayakshin et al. (2000) find that in certain conditions $\mathrm{Fe} \mathrm{K} \alpha$ photons can nevertheless be emitted: if the incident spectrum is steep $(\Gamma \gtrsim 2)$ or if the intrinsic disk flux exceeds the X-ray illuminating flux, in these cases the reflecting layer is expected to yield ionized iron emission lines. Ballantyne et al. (2001) show that the constant density models of Ross \& Fabian (1993) actually often give accurate fits to their "hydrostatic" reflection spectra, both statistically and parametrically, assuming an ASCA-like spectral resolution. On the other hand, the XSPEC pexriv and pexrav models give only poor fits to their spectra.

Whereas the value of the Fe $\mathrm{K} \alpha$ line energy, as measured by ASCA and BeppoSAX in MR 2251-178, clearly excludes neutral $\mathrm{Fe}$, the case for ionized reflection is less firm. The data strongly suggest that little reflection is present and that we are in fact mainly observing the direct ionizing continuum since very good fits are obtained with an exponentially cut-off power-law $\left(\chi_{\nu}^{2}=\right.$ 0.94, d.o.f. $=155$ ) or a pexriv or RFY99 model allowing the direct component to "shine" through at more than $80 \%\left(\chi_{\nu}^{2}=0.89\right.$, d.o.f. $=153$ with pexriv, see also Table 1). Because of the weak contribution of the reflection component the shape of the cut-off at $\sim 100 \mathrm{keV}$ must be intrinsic to the primary X-ray emission. However, despite its weak contribution, an ionized reflector can produce the observed ionized Fe emission, as shown with the RFY99 fit.

Broad band observations of Sy1 galaxies with BeppoSAX have revealed exponential cut-off energies $\lesssim 100 \mathrm{keV}$ in only a couple Sy1s: e.g. NGC 5548 $\left(E_{\text {cut-off }}=115_{-27}^{+39} \mathrm{keV}\right.$, Nicastro et al. 2000) and NGC 4151 (70 keV, Piro et al. 1998). Whereas in other Sy1s the cut-off occurs at much higher values, with lower limits around 200-300 keV (cf. Gondek et al. 1996; Matt 1998). More recently, Zdziarski et al. (2000) have measured the average OSSE spectra of Sy 1 and Sy 2. Using a cut-off power-law they derived expontenial cut-off energies of $120_{-60}^{+220}$ and $130_{-50}^{+220} \mathrm{keV}$ for Sy 1 and Sy $2 \mathrm{~s}$, respectively. It appears therefore that the cut-off energy measured by BeppoSAX for the RQ QSO MR 2251-178 is compatible with the average OSSE Sy 1 value as well as the BeppoSAX values obtained so far for Sy 1s.

\section{Conclusion}

We have for the first time observed MR 2251-178 in the 0.1-200 keV energy range using BeppoSAX. The spectral fitting described above shows that this source possibly combines two distinct signatures of ionized reflection which, to our knowledge, have not been detected together before in an individual radio quiet AGN: a strongly ionized $\mathrm{Fe} \mathrm{K} \alpha$ emission line and a contribution, in this case relatively weak, from an ionized reflection continuum. A spectral break is apparent at higher energies which can be parametrized with the help of an e-folded power-law. The cut-off energy is measured to be in the range $\sim 80$ $190 \mathrm{keV}$ (90\% confidence interval) and is compatible with values found for Sy 1 galaxies.

Acknowledgements. The BeppoSAX satellite is a joint ItalianDutch programme. AO acknowledges a Fellowship of the Swiss National Science Foundation during part of this work. She expresses gratitude to Prof. R. Staubert for having hosted her at the IAA in Tübingen during this period and wishes to thank J. Wilms and other colleagues form the IAAT for fruitful discussions about MR 2251-178. AO is very thankful to Prof. Netzer for allowing to use his ION98 model and giving valuable advice about the warm absorber fitting.

\section{References}

Anders, E., \& Grevesse, N. 1989, Geochimica et Cosmochimica Acta, 53, 197

Antonucci, R. 1993, ARAA, 31, 473

Ballantyne, D., Ross, R., \& Fabian, A. 2001, MNRAS, accepted 
Basko, M., Sunyaev, R., \& Titarchuk L. 1974, A\&A, 31, 249

Boella, G., Chiappetti, L., Conti, G., et al. 1997, A\&AS, 122, 327

Brunner, H., Müller, C., Friedrich, P., et al. 1997, A\&A, 326, 885

Fabian, A., Iwazawa, K., Reynolds, C., \& Young, A. 2000, PASP, 112, 1145

Fiore, F., Guainazzi, M., \& Grandi, P. 1999, Cookbook for BeppoSAX NFI Spectral Analysis, Version 1.2, BeppoSAX Science DATA Center

Frontera, F., Costa, E., Dal Fiume, D., et al. 1997, A\&AS, 122, 371

George, I., Turner, T., Netzer, H., et al. 1998, ApJS, 114, 73

George, I., Turner, T., Yaqoob, T., et al. 2000, ApJ, 531, 52

Gondek, D., Zdziarski, A., Johnson, W., et al. 1996, MNRAS, 282,646

Guainazzi, M., \& Matteuzzi, A. 1997, BeppoSAX Science Data Center Technical Report TR-011

Halpern, J. 1984, ApJ, 281, 90

Iwasawa, K., \& Taniguchi, Y. 1993, ApJ, 413, L15

Lawson, A., \& Turner, M. 1997, MNRAS, 288, 920

Maisack, M., Collmar, W., Barr, P., et al. 1995, A\&A, 298, 400

Manzo, G., Guarrusso, S., Santangelo, A., et al. 1997, A\&AS, 122,341

Matt, G. 1998, Nuclear Physics B, Proc. Suppl., 69/1-3, 467

Mineo, T., \& Stewart, G. 1993, MNRAS, 262, 817

Monier, E., Mathur, S., Wilkes, B., \& Elvis, M., ApJ, submitted [astro-ph/0102348]

Morisson, D., \& McCammon, D. 1983, ApJ, 270, 119

Nandra, K., \& Pounds, K. 1994, MNRAS, 268, 405

Nandra, K., George, I., Mushotzky, R., Turner, T., \& Yaqoob, T. 1997a, ApJ, 477, 602
Nandra, K., George, I., Mushotzky, R., Turner, T., \& Yaqoob, T. 1997b, ApJ, 488, L91

Nayakshin, S., Kazanas, D., \& Kallman, T. 2000, ApJ, 537, 833

Netzer, H. 1996, ApJ, 473, 781

Nicastro, F., Fiore, F., Perola, G., \& Elvis, M. 1999, ApJ, 512, 184

Nicastro, F., Piro, L., De Rosa, A., et al. 2000, ApJ, 536, 718

Orr, A., Parmar, A., Barr, P., \& Guainazzi, M. 2000, Adv. Space Res., 25(3/4), 459

Otani, C., Kii, T., \& Miya, K. 1997, in Proc. of IAU Symp. 188, The Hot Universe, ed. K. Koyama et al. (Kyoto, Japan)

Pan, H., Stewart, G., \& Pounds, K. 1990, MNRAS, 242, 177

Parmar, A. N., Martin, D. D. E., Bavdaz, M., et al. 1997, A\&AS, 122, 309

Parmar, A. N., Oosterbroek, T., Orr, A., et al. 1999, A\&AS, 136,407

Piro, L., Nicastro, F., Feroci, M., et al. 1998, Nucl. Phys. B, Proc. Suppl., 69/1-3, 481

Piro, L., De Rosa, A., Dadina, M., et al. 2000, Adv. Space Res., $25(3 / 4), 453$

Reeves, J., Turner, M., Ohashi, T., \& Kii, T. 1997, MNRAS, 292, 468

Reeves, J., \& Turner, M. 2000, MNRAS, 316, 234

Ross, R., \& Fabian, A. 1993, MNRAS, 261, 74

Ross, R., Fabian, A., \& Young, A. 1999, MNRAS, 306, 461

Staubert, R., \& Maisack, M. 1996, A\&A, 305, L41

Vignali, C., Comastri, A., Cappi, M., et al. 1999, ApJ, 516, 582

Williams, O., Turner, M., Stewart, G., et al. 1990, ApJ, 389, 157

Zdziarski, A., Poutanen, J., Johnson, W. N., et al. 2000, ApJ, 542,703 